\title{
Influência do sexo de indivíduos da mesma colônia no controle químico das populações do morcego hematófago Desmodus rotundus (Phyllostomidae) no Estado de São Paulo ${ }^{1}$
}

\author{
Murilo Novaes Gomes ${ }^{2 *}$, Wilson Uieda ${ }^{3}$ e Maria do Rosário \\ Dias de Oliveira Latorre ${ }^{4}$
}

\begin{abstract}
Gomes M., Uieda W. \& Latorre M.R.D.O. 2006. [Influence of sex differences in the same colony for chemical control of vampire Desmodus rotundus (Phyllostomidae) populations in the state of São Paulo, Brazil.] Influência do sexo de indivíduos da mesma colônia no controle químico das populações do morcego hematófago Desmodus rotundus (Phyllstomidae) no Estado de São Paulo. Pesquisa Veterinária Brasileira 26(1):38-43. Coordenadoria de Defesa Agropecuária/SP, Regional Mogi das Cruzes, Av. Cândido X.A.Sousa 35, Mogi das Cruzes, SP 08780-210, Brazil. Email: murilong@usp.br

The aim of this study was to verify the fidelity to the diurnal roosts by the genders of the vampire bat Desmodus rotundus, and to analyse the Warfarin gel $2 \%$ effectiveness for reduction of the size of vampire bat colonies, when individuals of different sexes were treated. In 1999 and 2000, 626 vampire bats were mist netted while leaving at night 12 diurnal roosts in the state of São Paulo. They were marked, and $10 \%$ of the roost population received the Warfarin gel $2 \%$ treatment. In Test I only males were treated, in Test II only females were treated, and in Test III 5\% of males and females were treated. After 5 and 10 days, the dead and the survivors were counted. Females were more loyal to the roosts $(p<0,01)$ and better wide spreader of the Warfarin gel $(p<0,01)$.
\end{abstract}

INDEX TERMS: Rabies, vampire bats, Desmodus rotundus, Warfarin gel.

RESUMO.- Os objetivos do presente trabalho foram estudar a fidelidade ao abrigo diurno por indivíduos de ambos os sexos da espécie Desmodus rotundus e verificar a eficácia da ação da pasta vampiricida $2 \%$ na redução do tamanho de suas colônias no estado de São Paulo, por meio de estudo experimental de campo. Durante os anos de 1999 e 2000, 626 morcegos distribuídos em 12 abrigos foram capturados com redes-de-espera (armadas durante a noite) e marcados. Em seguida, $10 \%$ da população previamente estimada recebeu a pasta vampiricida. No Experimento I foram tratados apenas machos, no Experimento II apenas fềmeas e no Experimento III, 5\% dos machos e 5\% das fêmeas foram tratados. Após 5 e 10 dias, foram feitas contagens dos morcegos que sobreviveram e morreram. As fêmeas mostraram-

\footnotetext{
${ }^{1}$ Recebido em 9 de setembro de 2005.

Aceito para publicação em 26 de outubro de 2005

${ }^{2}$ Coordenadoria de Defesa Agropecuária/SP, Regional Mogi das Cruzes Av. Cândido X.A.Sousa 35, Mogi das Cruzes, SP 08780-210. * Autor para correspondência. E-mail: murilong@usp.br

${ }^{3}$ Depto Zoologia, Instituto de Biociências, Unesp-Botucatu, Botucatu, SP 18618-000. E-mail: wuieda@ibb.unesp.br

${ }^{4}$ Depto Epidemiologia, Faculdade de Saúde Pública, USP, Av. Dr. Arnaldo 715, São Paulo, SP 01246-904.
}

se mais fiéis aos abrigos $(\mathrm{p}<0,01)$ e melhores disseminadoras de pasta vampiricida $(\mathrm{p}<0,01)$.

TERMOS DE INDEXAÇÃO: Raiva, morcego vampiro, Desmodus rotundus, Warfarina.

\section{INTRODUÇÃO}

Devido aos seus hábitos alimentares, aos prejuízos que têm causado à pecuária e aos problemas de saúde pública pela transmissão da raiva, o morcego hematófago Desmodus rotundus (E. Geoffroy 1810) tem recebido atenção dos pesquisadores e dos governos dos países da América Latina (Uieda 1987). Estudos biológicos, ecológicos e etológicos visando conhecer essa espécie no intuito de desenvolver métodos eficientes e viáveis de controle de suas populações começaram a ser realizados. Podem ser citados, como exemplos, os trabalhos de Linhart et al. (1972), Piccinini et al. (1986), Uieda (1987), Wilkinson (1988), Taddei et al. (1991), Massad et al. (2001) e Almeida et al. (2002).

Como é comum em mamíferos gregários, a espécie $D$. rotundus apresenta estrutura social caracterizada por hierarquia de dominância com formação de haréns (Wilkinson 1990), sendo que seus abrigos podem conter desde indivíduos solitários até aproximadamente 2.000 , no entanto, a maioria das colônias 
contém entre 20 e 100 morcegos (Uieda 1987). Uma importante característica dessa espécie é o intenso contato corporal dos indivíduos nas colônias, que se posicionam lado a lado e/ou um sobre outro, formando um grupo compacto, além do fato de realizarem limpeza mútua e regurgitação de alimento entre os membros do grupo (Linhart et al. 1972).

Esses comportamentos sugeriram a aplicação tópica de uma substância tóxica de ação lenta em alguns morcegos capturados para, após soltos, ao retornarem aos seus agrupamentos, contaminarem e levarem à morte os demais membros da colônia (Linhart et al. 1972). Esses autores usaram, em princípio, a clorofacionona e difenadiona como substância tóxica, e posteriormente, a Warfarina como terceiro anticoagulante testado para o controle da população desses morcegos. Tal componente químico, por ser mais barato e menos tóxico ao ambiente, é amplamente utilizado por equipes de controle da raiva dos herbívoros domésticos dos serviços de defesa agropecuária do país.

No final da década de 80 no Panamá, Wilkinson (1988) verificou que os comportamentos observados por Linhart et al. (1972), determinantes do uso de anticoagulantes para o controle de populações de $D$. rotundus, não eram iguais para todos os indivíduos de uma colônia. Os autores observaram que havia, principalmente, uma diferenciação comportamental entre indivíduos machos e fềmeas.

De um modo geral, Wilkinson (1990) observou que os machos adultos não eram limpos por outros morcegos, ao contrário das fêmeas e dos jovens (independentemente do sexo) que, na maior parte das vezes, participavam da limpeza em grupo. Um comportamento diferenciado também foi observado na partilha de alimento pois a regurgitação foi verificada, geralmente, nas relações entre mães e filhotes e entre as fêmeas adultas. Os machos adultos raramente foram relacionados a esse ritual, mas mais freqüentemente aos comportamentos agonísticos (situação em que o vírus rábico poderia ser transmitido) com os demais machos da colônia. $\mathrm{O}$ autor ainda revelou que um intenso contato corporal era realizado apenas por fêmeas e jovens concentrados em um grupo principal de uma colônia atendido por um macho dominante, com os machos subalternos circundando-os perifericamente. Observações semelhantes foram relatadas por Lord (1992) no nordeste da Argentina e Gomes \& Uieda (2004) no estado de São Paulo.

Este estudo parte da hipótese que o comportamento diferenciado entre machos e fêmeas, observado por Wilkinson (1988), poderia interferir nos métodos de controle desenvolvidos por Linhart et al. (1972). Assim, este estudo objetivou: 1) analisar a fidelidade ao abrigo diurno de indivíduos machos e fêmeas da espécie $D$. rotundus e 2) verificar a eficácia da ação da pasta vampiricida $2 \%$ na redução do tamanho de colônias do estado de São Paulo, quando a pasta foi aplicada em indivíduos machos, em fêmeas e em ambos os sexos.

\section{MATERIAL E MÉTODOS}

Com o intuito de contemplar os objetivos deste trabalho, 67 abrigos de morcegos nas diversas regiões do estado de São Paulo foram visitados com o auxílio das equipes de controle da raiva dos herbívoros domésticos da (CDA) Coordenadoria de Defesa Agropecuária da Secretaria da Agricultura e Abastecimento do Estado de São Paulo nos anos de 1999 e 2000. No entanto, somente 12 deles foram escolhidos por características como o acesso, se possuíam poucas saídas e se o tamanho das colônias era similar, isto é, se elas tinham um número próximo de indivíduos para permitir comparação posterior. O nome desses abrigos foi dado de acordo com seu tipo e os municípios onde estavam localizados. Maiores informações a respeito da metodologia da capturas dos morcegos, das características de cada abrigo (Fig. 1) e de suas respectivas colônias, podem ser verificadas em Gomes \& Uieda (2004).

Neste estudo, foi considerada como colônia os morcegos da mesma espécie, que viviam em um mesmo abrigo diurno, de ambos os sexos, em diferentes estágios de desenvolvimento e reprodutivo. Para análise da fidelidade dos indivíduos e eficácia da ação da pasta vampiricida foram considerados apenas os morcegos capturados quando estavam saindo do abrigo no dia da captura, perfazendo um total de 626 espécimes.

Em todas as viagens de visita aos abrigos diurnos de D. rotundus, foram utilizados os materiais comuns às noites de capturas de morcegos como pasta Vampiricida Vallé ${ }^{\circledR}$ (base de Warfarina $2 \%$ ),

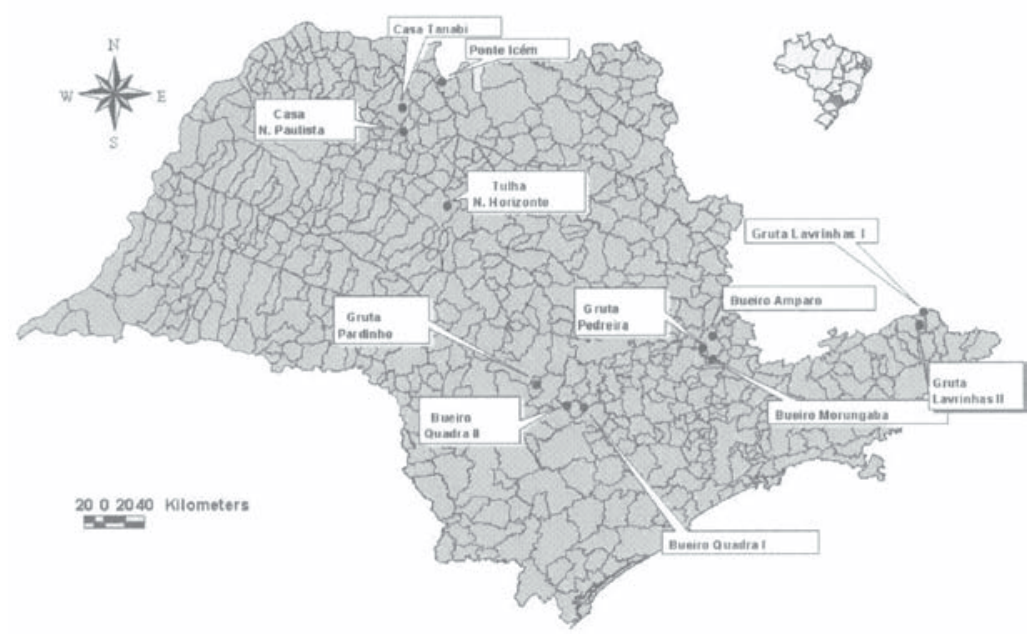

Fig.1. Localização no Estado de São Paulo dos abrigos diurnos de Desmodus rotundus durante experimento em 1999 e 2000. 
redes-de-espera de nylon para capturar quirópteros, lanternas, equipamentos de proteção individual (máscaras, luvas de raspa-decouro, capacete, macacão, galochas) e sacos de pano numerados para contenção, entre outros.

Nesta investigação, os morcegos que receberam a pasta de Warfarina $2 \%$ aplicada em seu dorso foram denominados de "tratados". Todas as carcaças de Desmodus rotundus encontradas no interior dos abrigos diurnos, durante o experimento, foram consideradas mortes ocorridas em consequiência da ação da pasta vampiricida.

\section{Desenho experimental}

Uma estimativa do número de indivíduos de cada abrigo diurno foi realizada cuidadosamente no início da tarde no primeiro dia de trabalho por meio da observação da quantidade de morcegos, suas fezes e movimentos da espécie dentro do abrigo. À noite, redesde-espera ("mist nets") foram armadas na saída do abrigo para a captura e em seguida, uma última avaliação foi feita após as 23:00 h, contando novamente os morcegos que se encontravam no interior do abrigo.

As sessões de captura noturna dos morcegos foram programadas na época das luas minguante e nova quando pelo menos a primeira parte da noite permanecia escura. Redes-de-espera de seis metros de comprimento foram armadas na(s) saída(s) dos abrigos por volta das 17:00 h e desarmadas às 23:00 h (o horário de verão foi desconsiderado). Com tais procedimentos, foram obstruídas a(s) saída(s) e acredita-se ter capturado, em média, $40 \%$ dos indivíduos da colônia avaliada inicialmente (o restante dos morcegos não havia saído de seus abrigos até às 23:00 h).

Após serem retirados da rede, os morcegos foram colocados individualmente em sacos de pano numerados. Ao final de cada sessão de captura, as redes foram recolhidas e foram passados cerca de dois gramas da pasta vampiricida no dorso de cada morcego com o auxílio de uma espátula de madeira, como habitualmente tem sido empregado pelos técnicos da área. Para efeito de controle, os morcegos tratados tiveram a unha de um dedo do pé direito e outra do pé esquerdo cortadas. Wilson Uieda (Comunicação pessoal) utilizou este método de marcação em um agrupamento de $D$. rotundus em cativeiro e não observou o crescimento das unhas cortadas por três meses. Os demais indivíduos da colônia capturados e soltos sem tratamento tiveram a unha de apenas um dedo esquerdo cortada.

Morcegos capturados pelo lado externo da rede (aparentemente estariam entrando no abrigo) tiveram duas unhas dos dedos esquerdos cortadas. Deste modo, pode-se identificar, posteriormente, os indivíduos e as carcaças de $D$. rotundus eliminados pela pasta. Morcegos sem unhas cortadas poderiam ser adições novas ao agrupamento após o tratamento ou indivíduos que não tinham sido capturados anteriormente por não terem saído até as 23 horas.

Após captura, tratamento e liberação dos morcegos, o abrigo foi visitado no $5^{\circ}$ e $10^{\circ}$ dias após a captura e tratamento. Nessas visitas, foi realizada a contagem de carcaças dos morcegos mortos e daqueles aparentemente sadios. Na noite do $10^{\circ}$ dia, a rede foi estendida novamente na saída do abrigo para contagem e estimativa dos indivíduos que sobraram e avaliação dos resultados.

Para cumprir o objetivo do trabalho, a pasta vampiricida foi usada da seguinte maneira:

a) Em $10 \%$ dos indivíduos machos adultos e sexualmente ativos de quatro colônias contendo indivíduos de ambos os sexos (Experimento I).

b) Em $10 \%$ dos indivíduos fêmeas adultas não grávidas e não lactantes de quatro colônias contendo indivíduos de ambos os sexos (Experimento II). c) Em 5\% dos indivíduos fêmeas adultas não grávidas e não lactantes e 5\% dos machos adultos ativos sexualmente de quatro colônias contendo indivíduos de ambos os sexos (Experimento III)

\section{Análises dos dados}

A fidelidade $\operatorname{dos} D$. rotundus aos abrigos diurnos foi verificada por meio de duas razões: 1) A razão dos morcegos machos tratados recuperados, por todos os machos tratados no primeiro dia do experimento, e a razão dos morcegos fêmeas tratadas recuperadas, por todas as fêmeas tratadas no primeiro dia do experimento; e 2) A razão do número de carcaças e sobreviventes machos marcados recuperados, por todos os machos marcados no primeiro dia do experimento, e a razão do número de carcaças e sobreviventes fêmeas marcadas recuperadas, por todas as fêmeas marcadas no primeiro dia do experimento.

Para caracterizar a eficácia da ação (Ev) da pasta vampiricida $2 \%$ sobre as colônias de morcegos hematófagos, foi calculada a proporção das carcaças recuperadas dos indivíduos mortos excluindo os tratados recuperados, pelas carcaças recuperadas dos indivíduos mortos, nos abrigos em que foram tratadas as fêmeas, nos abrigos em que foram tratados os machos e nos abrigos em que ambos os sexos foram tratados. A eficácia expressa em porcentagem pode ser determinada pela equação:

$$
\begin{aligned}
& E v=[(\beta-\xi) / \beta] \times 100, \text { onde: } \\
& E v=\text { Eficácia da ação da pasta vampiricida, } \\
& \beta=\text { Carcaças recuperadas, } \\
& \xi=\text { Carcaças recuperadas dos morcegos tratados. }
\end{aligned}
$$

Foi utilizado o programa EPIINFO 6.0 para o tratamento do banco de dados (Dean et al. 1994). Foram utilizados os testes de diferença de duas proporções para comparar a Ev entre os experimentos e o teste de associação pelo Qui-quadrado com correção de Yates para verificar a fidelidade ao abrigo diurno. Em todas as análises o valor de p aceito foi $<0,05$.

\section{RESULTADOS}

A primeira análise da diferença sexual da fidelidade dos morcegos aos abrigos diurnos analisados foi realizada apenas com as proporções das carcaças daqueles morcegos que foram tratados. Os resultados demonstraram que as carcaças das fêmeas tratadas foram significativamente mais recuperadas que as dos machos, em uma diferença de $17 \%(\mathrm{p}<0,01)$ (Quadro 1). A segunda análise realizada com as proporções das carcaças e dos sobreviventes marcados recuperados também demonstrou que as fêmeas foram significativamente mais recuperadas que os machos, em uma diferença de $13,8 \%(\mathrm{p}<0,01)$ (Quadro 2).

Os resultados obtidos nos Experimentos I, II e III foram de-

\begin{tabular}{|c|c|c|c|c|c|}
\hline \multirow[t]{3}{*}{ Sexo } & \multicolumn{4}{|c|}{ Recuperados } & \multirow{3}{*}{$\begin{array}{c}\text { Indivíduos } \\
\text { tratados }\end{array}$} \\
\hline & \multicolumn{2}{|c|}{ Sim } & \multicolumn{2}{|c|}{ Não } & \\
\hline & $\mathrm{N}$ & $\%$ & $\mathrm{~N}$ & $\%$ & \\
\hline Fêmeas & 38 & 44,1 & 48 & 55,8 & 86 \\
\hline Machos & 19 & 27,1 & 51 & 72,8 & 70 \\
\hline Total & 57 & 36,5 & 99 & 63,5 & 156 \\
\hline
\end{tabular}

Quadro 1. Carcaças de indivíduos machos e fêmeas de Desmodus rotundus tratados, recuperados ou não, em todos abrigos estudados, estado de São Paulo, 1999-2000

$p<0,01$ com correção de Yates. 
Quadro 2. Carcaças e indivíduos machos e fêmeas de Desmodus rotundus marcados, recuperados ou não, em todos abrigos estudados, no estado de São Paulo, 1999-2000

\begin{tabular}{cccccc}
\hline \multirow{2}{*}{ Sexo } & \multicolumn{4}{c}{ Recuperados } & \multirow{2}{*}{ Total } \\
\cline { 2 - 4 } & \multicolumn{2}{c}{ Sim } & \multicolumn{2}{c}{ Não } & \\
\cline { 2 - 4 } & $\mathrm{N}$ & $\%$ & $\mathrm{~N}$ & $\%$ & \\
\hline Fêmea & 180 & 49,7 & 182 & 50,3 & 362 \\
Macho & 95 & 35,9 & 169 & 64,1 & 264 \\
Total & 275 & 43,9 & 351 & 56,1 & 626 \\
\hline
\end{tabular}

$p<0,01$ com correção de Yates.

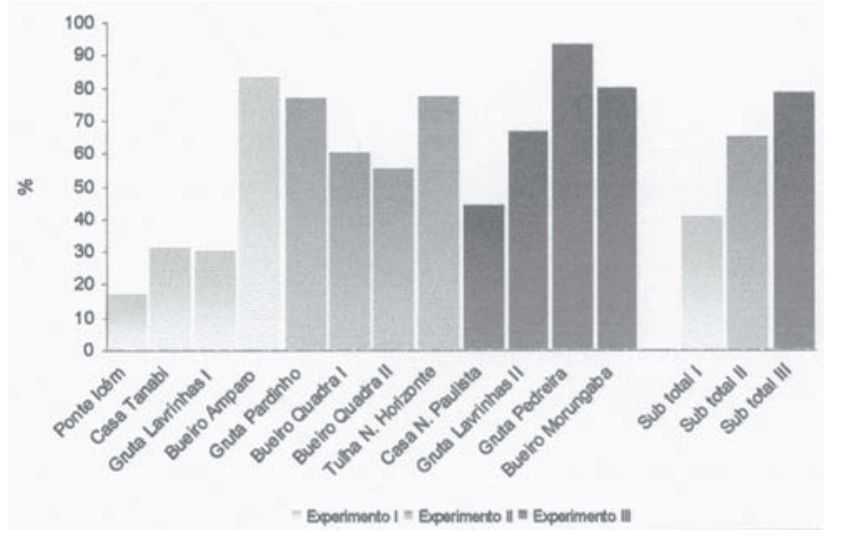

Fig.2. Redução do tamanho aproximado das colônias de Desmodus rotundus em cada abrigo estudado.

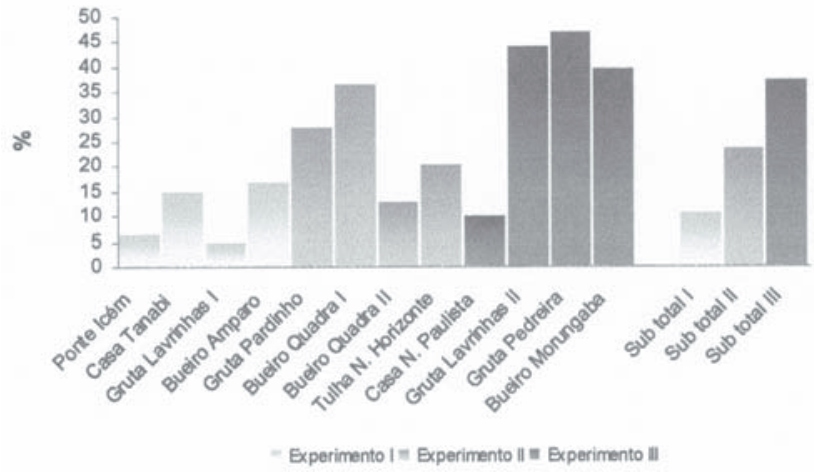

Fig.3. Redução, somente entre os marcados, do número de Desmodus rotundus em cada abrigo estudado.

monstrados pelas Figuras 2 e 3 e foi possível verificar que, no Experimento I, foi obtida, em média, uma redução de $40,4 \%$ no tamanho aproximado das colônias de morcegos. Por outro lado, na análise dos indivíduos marcados e recuperados, obteve-se $10,4 \%$ da redução do número de morcegos marcados no primeiro dia de captura. No Experimento II, ocorreu uma diminuição em média de $65 \%$ no tamanho aproximado das colônias de morcegos, porém, na análise dos indivíduos marcados e recuperados, observou-se $23,4 \%$ da redução do número de indivíduos. Por fim, no Experimento III foi verificada, em média, uma redução de $78,2 \%$. Já na análise dos indivíduos marcados e recupera-
Quadro 3. Número de morcegos tratados e não tratados mortos que foram recuperados em todos abrigos estudados no estado de São Paulo, em 1999-2000

\begin{tabular}{lcccc}
\hline \multicolumn{1}{c}{$\begin{array}{c}\text { Abrigos diurnos de } \\
\text { Desmodus rotundus } \\
\text { divididos por experimentos }\end{array}$} & $\begin{array}{c}\text { Morte por Warfarina } \\
\text { recuperados }\end{array}$ & Total & Ev \\
\cline { 2 - 4 } & Tratados & Não tratados & & \\
\hline Experimento I (Machos) & 13 & 12 & 25 & $48,0 \%$ \\
Experimento II (Fêmeas) & 29 & 163 & 192 & $84,9 \%$ \\
Experimento III (Ambos Sexos) & 15 & 113 & 128 & $88,3 \%$ \\
Total & 57 & 288 & 345 & $83,4 \%$
\end{tabular}

$\overline{p<0,01 \text { (entre I e II), } p=0,19}$ (entre II e III).

dos, obteve-se 37,0\% da redução do número de morcegos marcados no primeiro dia de captura.

A Ev expressa em porcentagem pode ser determinada pela equação apresentada anteriormente em Materiais e Métodos e o resultado alcançado foi apresentado pelo Quadro 3.

A comparação da Ev nos Experimentos II e III mostrou que não houve diferença estatisticamente significativa $(p=0,19)$ nos resultados de redução do número de indivíduos das colônias, ou seja, caso o uso da pasta vampiricida fosse realizado somente em indivíduos fêmeas ou em ambos os sexos, poder-se-ia considerar que os resultados seriam semelhantes na redução do tamanho das colônias de $D$. rotundus.

A comparação da Ev entre os Experimentos I e II mostrou que houve diferença estatisticamente significativa $(p<0,01)$ entre ambos. Assim, pôde-se considerar que tratando somente indivíduos fêmeas, o resultado esperado devesse ser maior na redução do tamanho das colônias de $D$. rotundus do que quando a pasta fosse utilizada apenas em indivíduos machos.

\section{DISCUSSÃO E CONCLUSÕES}

A recuperação de um maior número de carcaças de fêmeas tratadas e de indivíduos fềmeas marcados do que entre os machos pode ser uma indicação de maior fidelidade das fêmeas aos abrigos diurnos. Fidelidade semelhante foi observada por Trajano (1996) nas populações de Desmodus rotundus do Vale do Ribeira, sul do estado de São Paulo. A autora ressaltou que os deslocamentos entre abrigos de uma determinada área são realizados principalmente por indivíduos machos, sendo as fêmeas mais fiéis aos abrigos diurnos. Conforme Wilkinson (1990), os machos possuem comportamentos mais individualizados na colônia e dificilmente compartilham dos comportamentos grupais. Já as fêmeas são mais gregárias e, conseqüentemente, podem ser mais fiéis aos abrigos.

A maior fidelidade ao abrigo diurno por parte das fêmeas de D. rotundus pode interferir no resultado do controle químico de suas colônias devido ao seu maior tempo de permanência na colônia do respectivo abrigo e também a relações recíprocas com os demais membros. Assim sendo, as fêmeas tratadas parecem ser capazes de disseminar uma maior quantidade de pasta vampiricida entre os membros da colônia e, por conseguinte, levar a uma maior redução do número de morcegos. Por outro lado, os machos por serem menos fiéis aos refúgios, por não possuírem comportamento gregário e por estarem mais predispostos a mudanças de abrigos, parecem não ser bons dissemina- 
dores da pasta vampiricida, principalmente quando o macho que a recebeu não for um dominante.

$\mathrm{Na}$ análise da redução do tamanho estimado visualmente das colônias de $D$. rotundus, foi verificada uma maior redução no Experimento III, em que fêmeas e machos foram tratados $(78,2 \%)$. Uma redução intermediária (65\%) foi obtida no Experimento II, e por fim, o experimento de menor redução do número da colônia estimado foi o Experimento I, em que somente machos foram tratados, com uma porcentagem de 40,4.

No trabalho realizado por Moreira et al. (1980), em que os autores capturaram os morcegos com redes entomológicas no interior de 12 abrigos do estado de Minas Gerais e utilizaram pasta Warfarina $1 \%$ em $10 \%$ da população estimada visualmente, foi obtida uma redução global de $81,4 \%$ do tamanho inicial das colônias. Em dois trabalhos desenvolvidos por Piccinini et al. $(1977,1985)$ em uma gruta do estado de Pernambuco e outra no estado de Minas Gerais, obteve-se, respectivamente, uma redução de $98 \%$ (utilizando pasta de difenadiona) e $94,7 \%$ (pasta de Warfarina 1\%) no tamanho das colônias desses abrigos. Nesses últimos trabalhos, os autores capturaram os morcegos com auxílio de redes-de-espera armadas na saída da gruta durante o período noturno e o tamanho das colônias também foi estimado visualmente. Como no trabalho de Moreira et al. (1980), os de Piccinini et al. $(1977,1985)$ também não apresentaram dados sobre o sexo dos morcegos estudados.

De acordo com Crespo et al. (1961), Wimsatt (1969), Almeida et al. (2002) e Gomes \& Uieda (2004), a razão sexual das colônias de $D$. rotundus é de aproximadamente 1:1. Assim, acredita-se que na maioria das intervenções realizadas por Moreira et al. (1980) e Piccinini et al. $(1977,1985)$, a proporção de machos e fêmeas tratados deve ter sido similar a 1:1. Dessa forma, seria possível comparar seus resultados com aqueles obtidos no Experimento III do presente estudo.

O resultado obtido por Moreira et al. (1980) de $81,4 \%$ de redução é semelhante ao obtido no Experimento III $(78,2 \%)$. Essa pequena diferença pode ser conseqüência da concentração menor de Warfarina na pasta vampiricida. Uma menor concentração deve retardar o efeito deste anticoagulante sobre o organismo dos morcegos tratados, aumentando sua sobrevida, podendo contaminar assim um maior número de membros da colônia.

Os resultados obtidos por Piccinini et al. $(1977,1985)$ foram muito superiores ao obtido no Experimento III. A falta de repetições nesses experimentos e o uso de pastas vampiricidas de efeito mais prolongado podem ter levado a melhores resultados na redução das colônias de $D$. rotundus do que no Experimento III.

Na análise da redução da população de morcegos que foram marcados no primeiro dia de trabalho, foi verificada uma maior redução da população de morcegos no Experimento III, em que fêmeas e machos foram tratados $(37,0 \%)$, uma diminuição de 23,4\% foi obtida no Experimento II, e por fim, o experimento de menor redução dos indivíduos marcados foi o I, em que somente machos foram tratados, com uma porcentagem de 10,4.

Baseando-se nas observações de Wilkinson (1990) a respeito da distribuição espacial dos morcegos e composição sexual das colônias, os diferentes resultados obtidos neste estudo podem ter sido em conseqüência dos machos terem disseminado a pasta, principalmente, entre os membros periféricos da colônia, aqueles que não pertencem a grande massa de morcegos, e as fêmeas ao contrário, terem disseminado a pasta entre a maioria dos indivíduos do abrigo. No Experimento I, a média de redução da colônia foi menor que a média de redução no Experimento II, em conseqüência dos machos terem tido, aparentemente, menos contato com a maior parte dos outros indivíduos do que as fêmeas. No Experimento III, pode ter ocorrido a soma dos efeitos, ou seja, os machos que receberam a pasta podem ter eliminado os indivíduos periféricos e as fêmeas, os indivíduos que estavam relacionados diretamente ao grupo principal da colônia.

Realizando a análise da Ev nos três Experimentos, pode-se verificar que a diferença de proporções do experimento I e II foi de $36,9 \%$ e estatisticamente diferente, sugerindo que houve uma maior transferência da pasta vampiricida no Experimento II em que somente as fêmeas foram tratadas, do que no Experimento I, em que somente os machos foram tratados. Assim, indivíduos fêmeas parecem ter uma maior capacidade de disseminar pasta vampiricida pela colônia de um dado abrigo diurno. Comparando as duas proporções de Ev dos Experimentos II e III, verificase que a diferença de 3,6\% não foi estatisticamente significativa. Apesar dos dados mostrarem uma maior eficácia da ação da pasta no Experimento III, em que machos e fêmeas foram tratados, o resultado da análise estatística implica que o fato de se utilizar a pasta vampiricida $2 \%$ somente em fêmeas ou em ambos sexos pode levar a resultados semelhantes.

Esses diferentes papéis exercidos por fêmeas e machos de uma colônia de $D$. rotundus podem ser uma das causas de alguns insucessos no controle de redução de colônias realizado pelas equipes de controles da raiva. Como sugestão ao Programa Controle da Raiva dos Herbívoros, pode-se recomendar o uso da pasta vampiricida principalmente em indivíduos fêmeas como forma de reduzir drasticamente o tamanho das colônias dessa espécie hematófaga. No caso de captura de indivíduos machos, considerando que não são bons disseminadores da pasta vampiricida, parte poderia ser tratada e parte encaminhada para exame laboratorial de raiva para pesquisa da prevalência do vírus rábico.

Como os resultados do presente estudo sugerem que o método de uso da pasta vampiricida no dorso (espaço interescapular) dos morcegos elimina mais fêmeas do que machos e pelo fato de suas colônias terem em média uma razão sexual de 1:1 (Crespo et al. 1961, Wimsatt 1969, Almeida et al. 2002, Gomes \& Uieda 2004), pode-se esperar que um certo número de indivíduos machos sobre de cada colônia tratada. Esses indivíduos, provavelmente, manter-se-ão como grupos de machos solteiros e poderão se deslocar para outras áreas, conseguindo escapar do programa de controle da espécie e talvez podendo vir a interferir na propagação de uma epidemia de raiva em determinadas situações.

Fornes et al. (1974) e Lord (1988) relataram que a epidemia de raiva propaga-se de forma migratória, de acordo com os deslocamentos realizados pelos morcegos entre abrigos de uma região. Segundo Lord (1988), para a resolução de uma epidemia migratória, o controle das populações de morcegos deveria ser 
realizado à frente dos locais onde estão ocorrendo os focos de raiva em herbívoros domésticos e no rumo por onde a epidemia tenderia a percorrer. Caso o controle das populações seja realizado em regiões onde já esteja grassando a raiva, além de contradizer os ditames dos trabalhos de Fornes et al. (1974) e Lord (1988) e não resolvê-la efetivamente, as evidências deste trabalho demonstram que a epidemia poderia ser, ainda, mais dispersa por uma região. Considerando-se que os indivíduos machos em período de incubação da enfermidade teriam a chance de escapar do controle pelo método químico devido ao comportamento já descrito anteriormente, a enfermidade poderia se estender para as regiões onde eles estariam buscando novas colônias para viver, desde que as colônias que eles já estavam acostumados a freqüientar tivessem sido dizimadas.

Nessa condição, o método do uso de pasta no dorso dos morcegos poderia reduzir as colônias de $D$. rotundus, mas não seria capaz de controlar totalmente a raiva em herbívoros. De um modo geral, para reduzir a influência dos machos, recomenda-se também o uso da pasta vampiricida nas mordeduras alimentares em herbívoros provocadas pelos morcegos (método indireto). Tais mordeduras, além da sanguivoria, são realizadas por indivíduos de ambos os sexos, que deveriam, assim, ser eliminados igualmente.

Agradecimentos.- A todos os funcionários da CDA que participaram da elaboração dos experimentos de campo, para que este trabalho pudesse ser concretizado.

\section{REFERÊNCIAS}

Almeida E.O., Moreira E.C., Naveda L.A.B. \& Herrmann G.P. 2002. Combate ao Desmodus rotundus rotundus (E.Geoffroy, 1810) na região cárstica de Cordisburgo e Curvelo, Minas Gerais. Arq. Bras. Med. Vet. Zootec. 54(2):117-126.

Crespo J.A., Vanella J.M., Blood B.D. \& de Carlo J.M. 1961. Observaciones ecológicas del vampiro Desmodus rotundus en el norte de Córdoba. Revista del museo Argentino de Ciencias Naturales Bernadino Rivadavia. 4(6):131-160.

Dean, A.G., Dean, J.A., Coulombier, D., Brendel, K.A., Smith, D.C. \& Burton, A.H. 1994. Epi info, version 6: a word processing database and statistics program for epidemiology on microcomputers. Centers of Disease Control and Prevention, Atlanta. 589p.

Fornes A., Lord D.L., Kuns M.L., Larghi O.P., Fuenzalida E. \& Lazara L. 1974. Control of bovine rabies through vampire bat control. J. Wildlife Disease 10:310-316.

Gomes M.N. \& Uieda W. Abrigos diurnos, composição de colônias, dimorfismo sexual e reprodução do morcego hematófago Desmodus rotundus [E.Geoffrey] [Chiroptera, Phillostomidae] no Estado de São Paulo, Brasil. 2004. Revta Bras. Zoologia 21(3):629-638.

Linhart S.B., Flores Crespo R. \& Mitchell G.C. 1972. Control de murcielagos vampiros por medio de un anticoagulante. Boln Of. Sanit. Panam. 73(2):100-109.

Lord R.D. 1988. Control of vampire bats, p.215-226. In: Greenhall A.M. \& Schmidt U. (ed.) Natural History of Vampire Bats. CRC PRESS, Florida.

Lord R.D. 1992. Seasonal reproduction of vampire bats and its relation to seasonality of bovine rabies. J. Wildlife Disease 28(2): 292-294.

Massad E., Coutinho F.A.B., Burattini M.N., Sallum P.C. \& Lopez L.F. 2001. A Mixed Ectoparasite-Microparasite Model for Bat-Transmitted Rabies. Theoretical Population Biology. 60:265-279.

Moreira E.C., Silva J.A., Saturnino H.M. \& Viana F.C. 1980. O emprego da Warfarina no combate aos morcegos hematófagos. Arq. Esc. Vet. UFMG 32(3):383-392.

Piccinini R.S., Curvelo V.T., Cavalcanti R.J.G., Max A.J. \& Tabosa A.G. 1977. Resultados do controle de vampiros Desmodus rotundus rotundus em uma furna no Estado de Pernambuco, Brasil. Bolm Def. Sanit. Anim. 11(4):8592.

Piccinini R.S., Perachi A.L., Freitas C.E.A., Souza J.C.P. 1985. Vampiricidas de uso tópico em animais domésticos e em morcegos hematófagos. Pesq. Vet. Bras. 5(3):97-101.

Piccinini R.S., Perachi A.L., Albuquerque S.T., Raimundo S.D.L., Tannure A.M., Souza J.C.P. \& Furtado L.L. 1986. O uso do "Tecvampicid Pasta 1\% no controle de morcegos hematófagos Desmodus rotundus (Chiroptera). Pesq. Vet. Bras. 6(4):137-140.

Taddei A.V., Gonçalves C.A., Pedro W.A., Tadei W.J., Kotait I. \& Arieta, C. 1991. Distribuição do morcego vampiro Desmodus rotundus no Estado de São Paulo e a raiva dos animais domésticos. Coordenadoria de Assistência Técnica Integral, Campinas. 107p.

Trajano E. 1996. Movements of cave bats in southeastern Brazil, with emphasis on the population ecology of the common vampire bat, Desmodus rotundus. Biotropica 28(1):121-129.

Uieda W. 1987. Morcegos hematófago e a raiva dos herbívoros no Brasil. Anais do Seminário de Ciências da Faculdades Integradas de Uberaba, FIUBE, p.13-29.

Wilkinson G.S. 1985. The social organization of the common vampire bat. Behav. Ecol. Sociobiol. 7:123-134.

Wilkinson G.S. 1988. Social Organization and Behavior, p.85-95. In: Greenhall A.M. \& Schmidt U. (ed.) Natural History of Vampire Bats. CRC Press, Florida.

Wilkinson G.S. 1990. Food sharing in vampire bats. Scient. American 262(2):64-70.

Winsatt W.A. 1969. Transient behavior, nocturnal activity patterns, and feeding efficiency of vampire bats (Desmodus rotundus) under natural conditions. J. Mammalogy 50:233-44. 
O arquivo disponível sofreu correções conforme ERRATA publicada no Volume 26 Número 2 da revista. 